\title{
Article \\ Multiservice Loss Models in C-RAN Supporting Compound Poisson Traffic
}

\author{
Iskanter-Alexandros Chousainov ${ }^{1}\left(\mathbb{D}\right.$, Ioannis Moscholios ${ }^{1, * \mathbb{D}}$, Panagiotis Sarigiannidis ${ }^{2}$ (D) \\ and Michael Logothetis ${ }^{3}$ iD
}

1 Department of Informatics \& Telecommunications, University of Peloponnese, 22100 Tripolis, Greece; ichousain@uop.gr

2 Department of Electrical \& Computer Engineering, University of Western Macedonia, 50100 Kozani, Greece; psarigiannidis@uowm.gr

3 Department of Electrical \& Computer Engineering, University of Patras, 26504 Patras, Greece; mlogo@upatras.gr

* Correspondence: idm@uop.gr

Citation: Chousainov, I.-A.;

Moscholios, I.; Sarigiannidis, P.; Logothetis, M. Multiservice Loss Models in C-RAN Supporting Compound Poisson Traffic.

Electronics 2022, 11, 773. https:// doi.org/10.3390/electronics11050773

Academic Editors: Nurul I. Sarkar and Juan-Carlos Cano

Received: 6 February 2022

Accepted: 28 February 2022

Published: 2 March 2022

Publisher's Note: MDPI stays neutral with regard to jurisdictional claims in published maps and institutional affiliations.

Copyright: (C) 2022 by the authors. Licensee MDPI, Basel, Switzerland. This article is an open access article distributed under the terms and conditions of the Creative Commons Attribution (CC BY) license (https:// creativecommons.org/licenses/by/ $4.0 /)$.

\begin{abstract}
In this paper, a cloud radio access network (C-RAN) is considered where the baseband units form a pool of computational resource units (RUs) and are separated from the remote radio heads (RRHs). The RRHs are grouped into clusters based on their capacity in radio RUs. Each RRH serves different service-classes whose calls have different requirements in terms of radio and computational RUs and follow a compound Poisson process. This means that calls arrive in batches while each batch of calls follows a Poisson process. If the RUs' requirements of an arriving call are met, then the call is accepted in the serving RRH for an exponentially distributed service time. Otherwise, call blocking occurs. We initially start our analysis with a single-cluster C-RAN and model it as a multiservice loss system, prove that the model has a product form solution, and determine time and call congestion probabilities via a convolution algorithm. Furthermore, the previous model is extended to include the more complex case of many clusters of RRHs.
\end{abstract}

Keywords: cloud; radio access; call blocking; product form; convolution; compound Poisson; multiservice; cluster

\section{Introduction}

Based on the quite recent report of [1], it is estimated to have 4.4 billion fifth-generation (5G) subscriptions and a total global mobile data traffic of $288 \mathrm{~EB} /$ month by the end of 2027 (compared to $65 \mathrm{~EB} /$ month by the end of 2021). More than $60 \%$ of this traffic is expected to be carried by $5 \mathrm{G}$ networks, which are also anticipated to cover around $75 \%$ of the world's population in 2027. In such a demanding environment where wireless traffic increases and mobile users (MUs) require quite high data rates, extremely low latency and "anytime anywhere" connectivity, a wireless architecture such as the cloud radio access network (C-RAN) is regarded as being a quite promising 5G RAN solution [2,3].

The C-RAN architecture aims at warranting quality of service (QoS) to all users based on the following three components: (a) the remote radio head (RRH), which includes the antenna together with the radio frequency components and has the duty for the signal's transmission/reception, (b) the baseband units (BBUs), which form a BBU pool and are responsible for the baseband signal processing, and (c) a high-capacity fronthaul network, which connects the BBU pool with the RRHs via the common public radio interface (CPRI) [4-7].

In the recent past, various 5G C-RAN aspects have been studied, including: (a) functional splits, dimensioning and capacity issues of the fronthaul network [8-12], (b) privacy and security challenges as well as detailed frameworks for potential security attacks and threats [13-16], (c) energy and cost saving issues together with energy saving operating 
strategies [3,17-20] and (d) cooperation problems between different mobile network operators $[21,22]$. On the other hand, there exists a limited number of papers that investigate the quite interesting subject of MUs' call admission control in a 5G C-RAN and focus on proposing analytical algorithms (based on efficient and usually recursive formulas) for the computation of call blocking probabilities (CBP) [23-28]. Note that efficient CBP formulas are desirable by telecom engineers who have the network dimensioning responsibility.

In [23], the RRHs form a single cluster. By the term "single cluster" the authors of [23] consider the case that the capacity in all RRHs is the same and equal to $U$ radio resource units (RRUs). New calls arrive in this cluster following a Poisson process (PP) and constitute a single service-class since they have the same RRU and computational resource units (CRUs) requirements. Upon the call's arrival in an RRH, the call has two RU requirements: (1) a RRU from the RRH that will actually serve the call and (2) a CRU from the BBU. Call blocking occurs if either the RRU or the CRU is unavailable. Otherwise, the call is accepted in the serving RRH for a generally distributed service time. The steady-state probabilities in the model of [23] (named in our paper single-class single-cluster (SC-SC)) possess a product form solution (PFS). Such a solution is desirable to have in Markovian models since it leads to an accurate CBP determination (e.g., via recursive formulas or via algorithms based on successive convolutions). In [24,25], the case of overlapping cells has been included in the model of [23], while in [26] a generalization of [23] is considered based on the fact that the RRHs may form more than one clusters according to their RRU capacity. The model of [26] is named in our paper single-class multi-cluster (SC-MC). The SC-SC model has been also considered as the springboard for the models of [29,30]. In [29], instead of considering the case of PP traffic, the authors study the case of quasi-random traffic where a limited number of MUs have the duty to generate traffic. On the other hand, in [30], the C-RAN handles batches of calls that follow a PP [31,32]. Such an arrival process is important in traffic engineering since it can describe the case of bursty traffic. We name the model of [30] compound Poisson SC-SC (c-SC-SC). Finally, the model of [26] (i.e., the SC-MC model) has been analyzed in [33] under the assumption of quasi-random traffic as well as in [28] under the assumption of a variety of traffic sources (sources that generate compound Poisson traffic, Poisson traffic and quasi-random traffic).

The common characteristic of $[23-30,33]$ is that all MUs have the same RU requirements. More specifically, they require a single RRU (from the RRH that will serve them) and a single CRU from the BBU pool. However, in contemporary networks it is essential, for network planning, to consider a multiservice environment where MUs generate calls of various resource requirements [34-46]. In [45], we introduced two such multirate loss models for the C-RAN architecture and named them multi-class single-cluster and multiclass multi-cluster models (MC-SC and MC-MC, respectively). In [46], we extended both models by assuming that a finite number of MUs has the responsibility to generate traffic towards the RRHs. Recently, a new multirate loss model was proposed in [47] with the aim to recursively compute CBP in a C-RAN that serves Poisson traffic, via the classical Erlang Multirate Loss Model [48] and the retry loss models [49-51]. Although the model of [47] is interesting, it treats call blocking events due to lack of RRUs independent from call blocking events due to lack of CRUs. This assumption implies that the RRHs and the BBU are treated as two independent loss systems. Because of this, the models of [47] have the advantage that they simplify the necessary calculations (with the aid of classical recursive formulas) but may introduce significant error when compared to simulation.

In this paper, we extend the MC-SC and MC-MC models to include the significant (in terms of network dimensioning) scenario of having RRHs that are responsible for serving compound Poisson traffic. The idea of studying multiservice batch arrivals relies on the fact that such arrivals may well approximate the case of multiservice bursty traffic [30,52-58]. The proposed models are named herein compound Poisson multi-class single-cluster (c-MC-SC) and compound Poisson multi-class multi-cluster (c-MC-MC), respectively. Our contribution is as follows: (1) we propose the c-MC-SC model and show that it possesses a PFS, (2) we provide a brute force method as well as a convolution algorithm for the 
computation of various performance measures, including time congestion (TC) and call congestion (CC) probabilities, (3) we compare the TC probabilities results of the c-MC-SC model with the c-SC-SC model of [30], (4) we propose the c-MC-MC model and show that it possesses a PFS for the steady-state probability distribution and (5) we provide a brute force method as well as an algorithm based on successive convolutions for the determination of TC and CC probabilities.

The remainder of this paper is the following: In Section 2, we propose the c-MC-SC model. In Section 2.1, we present the PFS of the MC-SC model, while in Sections 2.2 and 2.3, we propose a brute force method and an algorithm based on successive convolutions for the TC and CC probabilities computation, respectively. In Section 3, we present an example where we compare the TC probability results of the proposed c-MC-SC model with those obtained via the c-SC-SC model of [30]. In Section 4, we propose the c-MC-MC model. In Section 4.1, we show that the c-MC-MC model possesses a PFS, while in Sections 4.2 and 4.3, we propose a brute force method and an algorithm based on successive convolutions for the TC and CC probabilities computation, respectively. Section 5 concludes this paper.

\section{The Proposed Compound Poisson MC-SC Model}

\subsection{The Analytical Model}

Consider the C-RAN of Figure 1, where the BBU, consisting of T CRUs, is separated from the $R$ RRHs. Each RRH has a capacity of $U$ RRUs and serves multiservice traffic. By the term "multiservice" we mean that incoming calls belong to particular service-classes based on their requirements in terms of RUs. More specifically, calls that have the same RUs requirements belong to the same service-class. Based on the abovementioned, a call that arrives at the $r$-th RRH $(r=1, \ldots, R)$ belongs to one of the $S_{r}$ different service-classes.

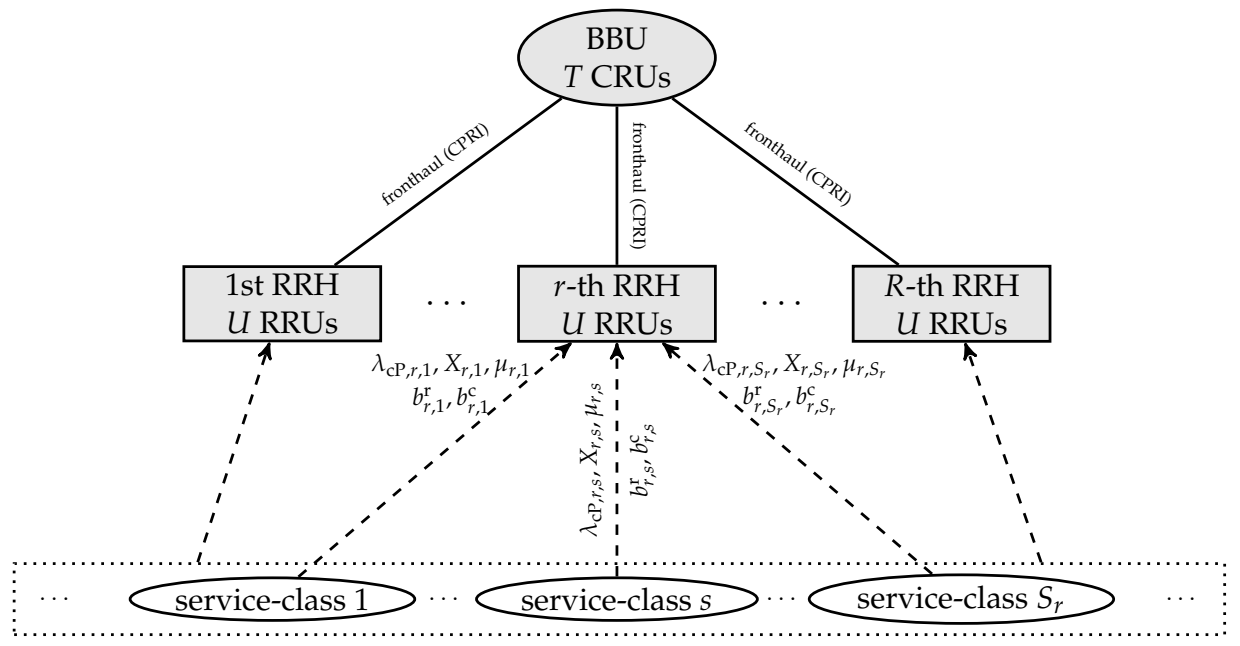

Figure 1. The proposed c-MC-SC model.

New service-class $r, s$ calls $\left(r=1, \ldots, R\right.$ and $\left.s=1, \ldots, S_{r}\right)$ arrive at the $r$-th RRH as batches via a compound PP having an arrival rate of $\lambda_{\mathrm{cP}, r, s}$. Let $X_{r, s}$ be the random variable that expresses the number of calls contained in a new batch, then $P\left(X_{r, s}=x\right)$ is the probability that $x$ calls are included in the new batch. Needless to say that if the value of $x$ is always equal to 1 call then the PP results. We assume that each of these $x$ calls does not depend on the remaining calls. This means that depending on the available CRUs and RRUs, one or more calls (of the same batch) can be accepted for service while the remaining can be blocked. An arriving call of service-class $r, s$ requires $b_{r, s}^{c}$ RRUs from the serving RRH (i.e., the $r$-th RRH) and $b_{r, s}^{\mathrm{c}}$ CRUs from the BBU. The call is accepted by the serving RRH if the necessary RUs are not occupied, i.e., if the $r$-th RRH has available $b_{r, s}^{\mathrm{r}}$ RRUs and the BBU has available $b_{r, s}^{\mathrm{c}}$ CRUs. When the system accepts the call, it serves it (via the $r$-th 
$\mathrm{RRH}$ ) for an exponentially distributed service time with mean $\mu_{r, s}^{-1}$. Assuming that there are no available RUs (either of radio or computational type), then call blocking occurs.

Let $n_{r, s}$ be the in-service calls of service-class $s$ in the $r$-th RRH. Then, the number of all in-service calls is expressed by the steady-state vector $\boldsymbol{n}=\left(n_{1,1}, \ldots, n_{1, S_{1}}, \ldots, n_{r, 1}, \ldots\right.$, $\left.n_{r, S_{r}}, \ldots, n_{R, 1}, \ldots, n_{R, S_{R}}\right)$. Let $\Omega$ be the system's state space given by:

$$
\boldsymbol{\Omega}=\left\{\boldsymbol{n}: n_{r, s} \geq 0, \quad \sum_{s=1}^{S_{r}} n_{r, s} b_{r, s}^{\mathrm{r}} \leq U, \quad \sum_{r=1}^{R} \sum_{s=1}^{S_{r}} n_{r, s} b_{r, s}^{\mathrm{c}} \leq T\right\} .
$$

In order to analyze the proposed c-MC-SC model, we focus on the steady-state of the model and show that the probability distribution $P_{\mathrm{cP}}(\boldsymbol{n})$ has a PFS. The latter can be derived based on the fact that local balance does exist between certain levels as the following analysis shows.

More specifically, for each state $\boldsymbol{n}$ let $L_{\boldsymbol{n}}^{(r, s)}$ be the level that separates $\boldsymbol{n}$ from $\boldsymbol{n}_{r, s}^{+}=$ $\left(n_{1,1}, \ldots, n_{1, S_{1}}, \ldots, n_{r, 1}, \ldots, n_{r, s}+1, \ldots, n_{r, S_{r}}, \ldots, n_{R, 1}, \ldots, n_{R, S_{R}}\right)$, see Figure 2 . To cross this level it is necessary to have a service-class $s$ batch arrival in the $r$-th RRH or a service-class $s$ call departure. The latter takes place when a call completes its service.

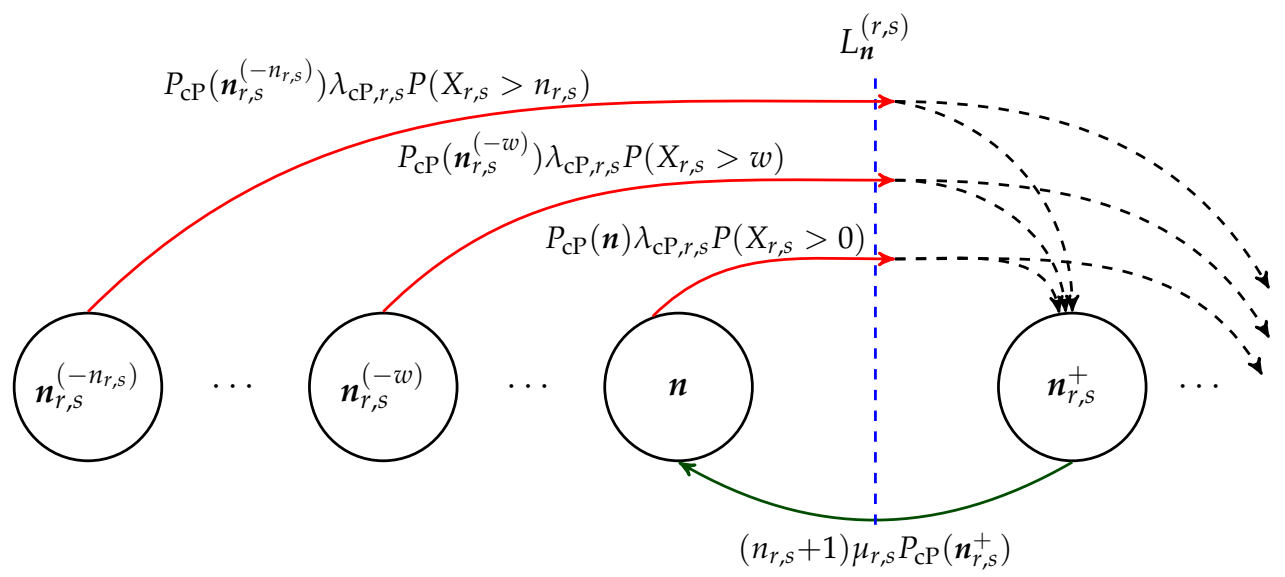

Figure 2. Local balance for the level $L_{n}^{(r, s)}$ of the c-MC-SC model.

We initially examine the case of a batch arrival. Then, the "upward" probability flow across $L_{n}^{(r, s)}$ is given by the following formula:

$$
p^{(\mathrm{upw})}\left(L_{n}^{(r, s)}\right)=\sum_{w=0}^{n_{r, s}} P_{\mathrm{cP}}\left(\boldsymbol{n}_{r, s}^{(-w)}\right) \lambda_{\mathrm{cP}, r, s} P\left(X_{r, s}>w\right)
$$

where $\quad \boldsymbol{n}_{r, s}^{(-w)}=\left(n_{1,1}, \ldots, n_{1, S_{1}}, \ldots, n_{r, 1}, \ldots, n_{r, s}-w, \ldots, n_{r, S_{r}}, \ldots, n_{R, 1}, \ldots, n_{R, S_{R}}\right)$ and $P_{\mathrm{cP}}\left(\boldsymbol{n}_{r, S}^{(-w)}\right)$ is the corresponding steady state probability.

Consider now the case of an in-service service-class $r, s$ call, which completes service. Then, the "downward" probability flow across $L_{n}^{(r, s)}$ is:

$$
p^{(\mathrm{dnw})}\left(L_{\boldsymbol{n}}^{(r, s)}\right)=\left(n_{r, s}+1\right) \mu_{r, s} P_{\mathrm{cP}}\left(\boldsymbol{n}_{r, s}^{+}\right)
$$

Via (2) and (3), we can write the following local balance equation across $L_{n}^{(r, s)}$ for service-class $r, s$ calls:

$$
\begin{aligned}
p^{(\mathrm{upw})}\left(L_{n}^{(r, s)}\right) & =p^{(\mathrm{dnw})}\left(L_{\boldsymbol{n}}^{(r, s)}\right) \text { or } \\
\sum_{w=0}^{n_{r, s}} P_{\mathrm{cP}}\left(\boldsymbol{n}_{r, s}^{(-w)}\right) \lambda_{\mathrm{cP}, r, s} P\left(X_{r, s}>w\right) & =\left(n_{r, s}+1\right) \mu_{r, s} P_{\mathrm{cP}}\left(\boldsymbol{n}_{r, s}^{+}\right) .
\end{aligned}
$$


Apart from (4), we also examine the corresponding global balance equation:

$$
p^{(\mathrm{upw})}\left(L_{\boldsymbol{n}_{r, s}^{-}}^{(r, s)}\right)+p^{(\mathrm{dnw})}\left(L_{n}^{(r, s)}\right)=p^{(\mathrm{upw})}\left(L_{n}^{(r, s)}\right)+p^{(\mathrm{dnw})}\left(L_{\boldsymbol{n}_{r, s}^{-}}^{(r, s)}\right),
$$

where $L_{\boldsymbol{n}_{r, s}^{-}}^{(r, s)}$ is the level that separates $\boldsymbol{n}_{r, s}^{-} \equiv \boldsymbol{n}_{r, s}^{(-1)}$ from $\boldsymbol{n}$.

The description of the four terms of (5) (from left to right) is as follows: (a) the first term refers to the "upward" probability flow into state $n$ due to an arrival of a batch, (b) the second term refers to the "downward" probability flow into $n$ due to a call departure, (c) the third term expresses the "upward" probability flow out of $n$ due to an arrival of a batch and (d) the last term expresses the "downward" probability flow out of $n$ due to a departure of a call.

Summing over $S_{r}$ in all RRHs leads to the global balance equation for $n$ :

$$
\sum_{r=1}^{R} \sum_{s=1}^{S_{r}}\left[p^{(\mathrm{upw})}\left(L_{\boldsymbol{n}_{r, s}^{-}}^{(r, s)}\right)+p^{(\mathrm{dnw})}\left(L_{\boldsymbol{n}}^{(r, s)}\right)\right]=\sum_{r=1}^{R} \sum_{s=1}^{S_{r}}\left[p^{(\mathrm{upw})}\left(L_{\boldsymbol{n}}^{(r, s)}\right)+p^{(\mathrm{dnw})}\left(L_{\boldsymbol{n}_{r, s}^{-}}^{(r, s)}\right)\right]
$$

Equations (5) and (6) can be satisfied by the PFS:

$$
P_{\mathrm{cP}}(\boldsymbol{n})=\frac{1}{N} \prod_{r=1}^{R} \prod_{s=1}^{S_{r}} P_{r, s}\left(n_{r, s}\right)
$$

where $N$ is the normalization constant, $N=\sum_{n \in \Omega} \prod_{r=1}^{R} \prod_{s=1}^{S_{r}} P_{r, s}\left(n_{r, s}\right)$ and

$$
P_{r, s}(n)=\left\{\begin{array}{ll}
\frac{\alpha_{\mathrm{CP}, r, s}}{n} \sum_{x=1}^{n} P_{r, s}(n-x) P\left(X_{r, s}>x-1\right), & \text { for } n \geq 1 \\
1, & \text { for } n=0
\end{array},\right.
$$

where $\alpha_{\mathrm{cP}, r, s}=\lambda_{\mathrm{cP}, r, s} / \mu_{r, s}$.

Having calculated $P_{\mathrm{cP}}(\boldsymbol{n})$, we compute the total TC probabilities $B_{\mathrm{tot}, r, s^{\prime}}^{\mathrm{TC}}$ as follows:

$$
B_{\mathrm{tot}, r, s}^{\mathrm{TC}}=B_{\mathrm{c}, r, s}^{\mathrm{TC}}+B_{\mathrm{r}, r, s}^{\mathrm{TC}}
$$

where $B_{\mathrm{c}, r, s}^{\mathrm{TC}}, B_{\mathrm{r}, r, s}^{\mathrm{TC}}$ refer to the TC events caused due to the lack of CRUs and RRUs, respectively.

Based on (7), we determine $B_{\mathrm{tot}, r, s}^{\mathrm{TC}}$ either via a brute-force method or via an algorithm based on successive convolutions (see Sections 2.2 and 2.3, respectively).

\subsection{The Brute Force Method}

Based on (7), the values of $B_{\mathrm{r}, r, S}^{\mathrm{TC}}$ can be determined via:

$$
B_{\mathrm{r}, r, S}^{\mathrm{TC}}=\sum_{\boldsymbol{n} \in \boldsymbol{\Omega}_{r, s}^{U,<T}} P_{\mathrm{CP}}(\boldsymbol{n}),
$$

where $\boldsymbol{\Omega}_{r, s}^{U,<T}=\left\{\boldsymbol{\Omega}_{r, s}^{U} \cap \boldsymbol{\Omega}_{r, s}^{<T}\right\}, \boldsymbol{\Omega}_{r, s}^{U}=\left\{\boldsymbol{n}: U-b_{r, s}^{\mathrm{r}}<\sum_{y=1}^{S_{r}} n_{r, y} b_{r, y}^{\mathrm{r}} \leq U\right\}, \boldsymbol{\Omega}_{r, s}^{<T}=\{\boldsymbol{n}:$ $\left.\sum_{x=1}^{R} \sum_{y=1}^{S_{x}} n_{x, y} b_{x, y}^{\mathrm{c}} \leq T-b_{r, s}^{\mathrm{c}}\right\}$.

Similarly, the values of $B_{\mathrm{c}, r, s}^{\mathrm{TC}}$ can be obtained via:

$$
B_{\mathrm{c}, r, S}^{\mathrm{TC}}=\sum_{n \in \boldsymbol{\Omega}_{r, T}^{=T}} P_{\mathrm{cP}}(\boldsymbol{n}),
$$

where $\boldsymbol{\Omega}_{r, s}^{=T}=\left\{\boldsymbol{n}: T-b_{r, s}^{\mathrm{c}}<\sum_{x=1}^{R} \sum_{y=1}^{S_{x}} n_{x, y} b_{x, y}^{\mathrm{c}} \leq T\right\}$. The set $\boldsymbol{\Omega}_{r, s}^{=T}$ includes two kinds of blocking states: those that express the CRUs unavailability and those that express both RRUs and CRUs unavailability. 
Equations (10) and (11) reveal that the determination of $B_{\mathrm{r}, r, S}^{\mathrm{TC}}$ and $B_{\mathrm{c}, r, S}^{\mathrm{TC}}$ is complex since it is mandatory to have enumeration/processing of $\Omega$. Thus, we suggest using this method only for tutorial examples.

\subsection{The Proposed Convolution Algorithm for the c-MC-SC Model}

This algorithm is based on the model's PFS and also requires that $b_{r, s}^{\mathrm{r}}=b_{r, s}^{\mathrm{c}}$, i.e., the RRUs requirement, should match the computational one. The following steps describe the algorithm:

\section{Step 1}

In this step, we consider each RRH and compute its occupancy distribution. To this end, we start by computing the occupancy distribution for each service-class $s$ of the $r$-th $\mathrm{RRH}, q_{r, s}(j)$, considering that only service-class $s$ calls exist in the RRH and assuming that $q_{r, s}(0)=1$ :

$$
q_{r, s}(j)=\frac{\alpha_{\mathrm{cP}, r, s}}{i} \sum_{x=1}^{i} q_{r, s}\left(j-x b_{r, s}^{\mathrm{r}}\right) P\left(X_{r, s}>x-1\right),
$$

where $i=j / b_{r, s}^{\mathrm{r}}$ expresses the service-class $s$ calls in the $r$-th RRH and $j \in\left\{i b_{r, s}^{\mathrm{r}}: i=\right.$ $\left.0, \ldots,\left\lfloor U / b_{r, s}^{\mathrm{r}}\right\rfloor\right\}$ expresses the corresponding occupied RRUs.

Next, we compute the aggregated occupancy distribution, $q_{r}$, of the $r$-th RRH, by successive convolution of all $q_{r, s}$ :

$$
q_{r}=q_{r, 1} * \ldots * q_{r, s} * \ldots * q_{r, S_{r}} .
$$

where the convolution operation between $q_{r, x} \equiv q_{a}$ and $q_{r, y} \equiv q_{b}$ defined on the domain $\{0, \ldots, U\}$ is given by:

$$
q_{a} * q_{b}=\left\{q_{a}(0) \cdot q_{b}(0), \sum_{x=0}^{1} q_{a}(x) \cdot q_{b}(1-x), \ldots, \sum_{x=0}^{U} q_{a}(x) \cdot q_{b}(U-x)\right\} .
$$

Finally, the normalized occupancy distribution of the $r$-th $\mathrm{RRH}, q_{r}^{\prime}$, is calculated via $q_{r}^{\prime}(j)=q_{r}(j) / N_{r}$ where $N_{r}=\sum_{j=0}^{U} q_{r}(j)$.

Step 2

Herein, we focus on the R RRHs, excluding the $r$-th one, and determine the corresponding aggregated occupancy distribution, via:

$$
Q_{\mathrm{cP},(-r)}=q_{1}^{\prime} * \ldots * q_{r-1}^{\prime} * q_{r+1}^{\prime} * \ldots * q_{R}^{\prime} .
$$

The convolution operation between $q_{x}^{\prime} \equiv q_{a}$ and $q_{y}^{\prime} \equiv q_{b}$, applied on the domain $\{0, \ldots, T\}$ is given by (14).

\section{Step 3}

Based on the previous two steps we now consider the (convolution) operation $\left(Q_{\mathrm{cP},(-1)} * q_{1}^{\prime}\right)(j)$ on the domain $j \in\{0, \ldots, T\}$. This operation results in the unnormalized values of the CRUs occupancy distribution $Q_{\mathrm{cP}}(j)$, which can be normalized via the constant $N_{\mathrm{CP}}^{*}=\sum_{j=0}^{T} Q_{\mathrm{CP}}(j)$, resulting in:

$$
Q_{\mathrm{cP}}^{\prime}(j)=\frac{Q_{\mathrm{cP}}(j)}{N_{\mathrm{cP}}^{*}} .
$$

To obtain $Q_{\mathrm{cP}}^{\prime}(j)$, we can focus on any of the $R$ RRHs since the convolution operation is commutative and associative. 
We continue by computing the TC probabilities due to lack of CRUs (see (17)) and RRUs (see (18)):

$$
\begin{gathered}
B_{\mathrm{c}, r, s}^{\mathrm{TC}}=\sum_{j=T-b_{r, s}^{\mathrm{c}}+1}^{T} Q_{\mathrm{cP}}^{\prime}(j), \\
B_{\mathrm{r}, r, \mathrm{~s}}^{\mathrm{TC}}=\frac{1}{N_{\mathrm{cP}}^{*}} \sum_{x=U-b_{r, s}^{\mathrm{r}}+1}^{U} q_{r}^{\prime}(x) \sum_{y=x}^{T-b_{r, s}^{\mathrm{r}}} Q_{\mathrm{cP},(-r)}\left(T-b_{r, s}^{\mathrm{r}}-y\right) .
\end{gathered}
$$

The values of CC probabilities for service-class $r, s$ calls, $B_{r, s}^{\mathrm{CC}}$, can be calculated via:

$$
B_{r, s}^{\mathrm{CC}}=\frac{\alpha_{\mathrm{CP}, r, s} \bar{X}_{r, s}-\bar{n}_{r, s}}{\alpha_{\mathrm{CP}, r, s} \bar{X}_{r, s}}
$$

where $\bar{X}_{r, s}$ is the average size of new batches of service class $s$ that arrive at the $r$-th RRH given by $\bar{X}_{r, s}=\sum_{x=1}^{\infty} x P\left(X_{r, s}=x\right)$, while $\bar{n}_{r, s}$ refers to the mean number of service-class $s$ calls serviced in the $r$-th RRH and can be calculated via:

$$
\bar{n}_{r, s}=\frac{1}{N_{\mathrm{cP}}^{*}} \sum_{j=1}^{U} y_{r, s}(j) q_{r}^{\prime}(j) \sum_{l=0}^{T-j} Q_{\mathrm{cP},(-r)}(l),
$$

where $y_{r, s}(j)$ expresses the average number of service-class $s$ calls in state $j$ of the $r$-th RRH computed via:

$$
y_{r, s}(j)=\frac{\alpha_{\mathrm{cP}, r, s}}{q_{r}^{\prime}(j)} \sum_{l=1}^{\left\lfloor j / b_{r, s}^{\mathrm{r}}\right\rfloor} q_{r}^{\prime}\left(j-l b_{r, s}^{\mathrm{r}}\right) P\left(X_{r, s}>l-1\right) .
$$

Regarding the distribution of the batch size, an important distribution is the geometric [32]. For that particular case, we have $P\left(X_{r, s}>x\right)=\beta_{r, s}^{x}$ and $\bar{X}_{r, s}=\left(1-\beta_{r, s}\right)^{-1}$, where $\beta_{r, s}$ is the distribution's parameter.

\section{Evaluation}

In this section, we present a C-RAN example and provide analytical TC probabilities results in the case of the proposed c-MC-SC model and the existing c-SC-SC model of [30]

We consider $R=6$ RRHs of capacity $U=10$ RRUs and let $T=30$ CRUs. The $r$-th RRH serves calls from $S_{r}$ service-classes and let $b_{r, s}=b_{r, s}^{\mathrm{c}}=b_{r, s}^{\mathrm{r}}$. More specifically, let $S_{1}=3$ be the service-classes of the 1 st RRH and assume that $b_{1,1}=1 \mathrm{RU}, b_{1,2}=2$ RUs and $b_{1,3}=3$ RUs. On the same hand, let $S_{2}=2$ be the service-classes of the 2 nd RRH and consider that $b_{2,1}=2$ RUs and $b_{2,2}=3$ RUs. Similarly, let $S_{3}=S_{4}=2$ and $S_{5}=S_{6}=1$

\begin{tabular}{|c|c|c|c|c|c|c|c|c|c|c|}
\hline \multirow{2}{*}{$\frac{R=6 \mathrm{RRH} ;}{r \text {-th RRH }\left(S_{r}\right)}$} & \multicolumn{10}{|c|}{$T=30$ CRUs. } \\
\hline & 1st & & & & & & & & 5 th (1) & 6th (1) \\
\hline service-class, $s$ & 2 & 3 & 1 & 2 & 1 & 2 & 1 & 2 & 1 & 1 \\
\hline band requirements (RUs), $b_{r, s}$ & 2 & 3 & 2 & 3 & 1 & 3 & 1 & 2 & 2 & 1 \\
\hline
\end{tabular}
be the service-classes of the $3 \mathrm{rd}$, the $4 \mathrm{th}$, the 5 th and the 6 th RRH. The corresponding RU values are the following: $b_{3,1}=1 \mathrm{RU}, b_{3,2}=3 \mathrm{RUs}, b_{4,1}=1 \mathrm{RU}, b_{4,2}=2 \mathrm{RUs}, b_{5,1}=2 \mathrm{RUs}$ and $b_{6,1}=1$ RU. Table 1 summarizes the C-RAN setup configuration.

Table 1. The setup of the C-RAN example.

Regarding the batch size, we consider that it follows the geometric distribution having a parameter of $\beta_{r, s}=0.2$ for each service-class $s$ in all RRHs $\left(r=1, \ldots, 6, s=1, \ldots, S_{r}\right)$. This means that, on average, an incoming batch contains $\bar{X}_{r, s}=\left(1-\beta_{r, s}\right)^{-1}=1.25$ calls.

The system serves a call for an exponentially distributed service time with parameter $\mu_{r, s}=1$. Let the arrival rate of batches be $\lambda_{\mathrm{cP}, r, s}=1$ and therefore $\alpha_{\mathrm{cP}, r, s}=\lambda_{\mathrm{cP}, r, s} / \mu_{r, s}=1$. We shall refer to $\alpha_{\mathrm{cP}, r, s}$ as the incoming traffic-load. We evaluate the network of our example 
considering TC probabilities for a total of 31 steps, where the arrival rate of the batches, $\lambda_{\mathrm{cP}, r, s}$, is increased in each step by 0.2 . The results of all steps are presented in Figures 3-9, where the $\mathrm{x}$-axis refers to the step number. Thus, at point 31 the value of $\alpha_{\mathrm{cP}, r, s}$ is 7.0 for all incoming batches in all RRH.

In the case of the single service c-SC-SC model, it is necessary to determine the quantity $\alpha_{\mathrm{cP}, r}$ for every RRH. Because of this, we define a load factor $l_{r}(r=1, \ldots, 6)$, expressed via $l_{r}=\sum_{r=1}^{S_{r}} b_{r, s}$ where $S_{r}$ and $b_{r, s}$ are the corresponding c-MC-SC values. Note that the initial values of $\alpha_{\mathrm{CP}, r}$ are equal to the corresponding value of $l_{r}$, i.e.,: $\left(\alpha_{\mathrm{cP}, 1}, \alpha_{\mathrm{cP}, 2}, \alpha_{\mathrm{cP}, 3}, \alpha_{\mathrm{cP}, 4}\right.$, $\left.\alpha_{\mathrm{CP}, 5}, \alpha_{\mathrm{CP}, 6}\right)=(6,5,4,3,2,1)$. As in the case of the c-MC-SC model, 31 steps are considered, where in each step $\alpha_{\mathrm{cP}, r}$ increases by $0.2 l_{r}$.

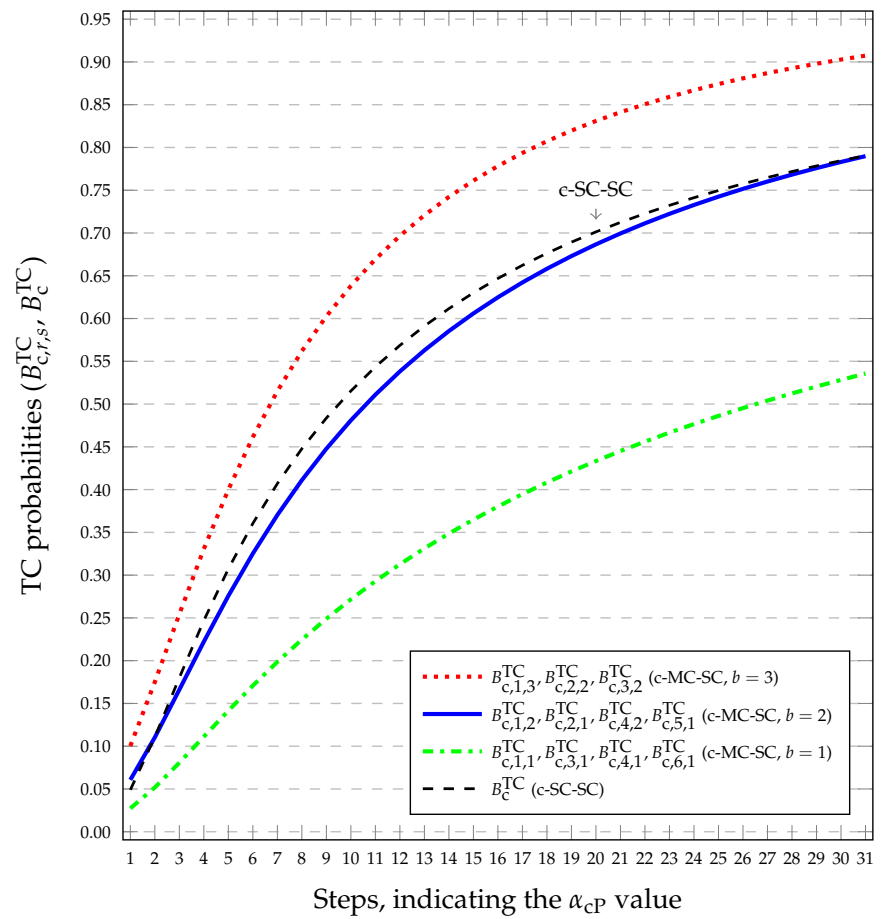

Figure 3. TC probabilities, $B_{\mathrm{c}, r, s}^{\mathrm{TC}}$, for all services-classes in all RRH (c-MC-SC vs. c-SC-SC).

In Figure 3, we present the TC probabilities results $\left(B_{\mathrm{c}, r, s}^{\mathrm{TC}}\right)$, due to a lack of CRUs, of the c-MC-SC model and the corresponding results $\left(B_{\mathrm{C}}^{\mathrm{TC}}\right)$ of [30]. Based on Figure 3, we conclude that: (a) the increase in incoming traffic-load increases TC probabilities and (c) the c-SC-SC model cannot provide TC probabilities close to the proposed model since the former refers to a single service model.

In Figures 4-6, the 1st (see Figure 4), the 2nd (see Figure 5) and the 3rd RRH (see Figure 6) are presented. More specifically, in Figure 4, we present the TC probabilities results of $B_{\mathrm{r}, 1, \mathrm{~T}}^{\mathrm{TC}}$ for the proposed model and the model of [30]. In the case of [30], points 1 and 31 in the $x$-axis of Figure 4 refer to $\alpha_{\mathrm{CP}, 1}=6$ and $\alpha_{\mathrm{CP}, 1}=42$, respectively. Similarly, in Figure 5, we present the TC probabilities results of $B_{\mathrm{r}, 2, S}^{\mathrm{TC}}$ for the proposed model and the model of [30]. In the case of [30], points 1 and 31 in the x-axis of Figure 5 refer to $\alpha_{\mathrm{CP}, 2}=5$ and $\alpha_{\mathrm{CP}, 2}=35$, respectively. Finally, in Figure 6, we present the TC probabilities results of $B_{\mathrm{r}, 3, s}^{\mathrm{TC}}$ for the proposed model and the model of [30]. In the case of [30], points 1 and 31 in the $\mathrm{x}$-axis of Figure 6 refer to $\alpha_{\mathrm{CP}, 3}=4$ and $\alpha_{\mathrm{CP}, 3}=28$, respectively. According to Figures 4-6, we observe that: (a) the TC probabilities of the c-SC-SC model cannot provide TC probabilities close to the proposed model and (c) the TC probabilities increase and then start to decrease as the incoming traffic-load increases. This behavior can be justified with the aid of Figure 3. According to Figure 3, the TC probabilities (due to the unavailability of CRUs) increase as the incoming traffic-load increases, and therefore, it is expected to 
have more RRUs available for new calls. The latter explains the TC probabilities decrease in Figure 6.

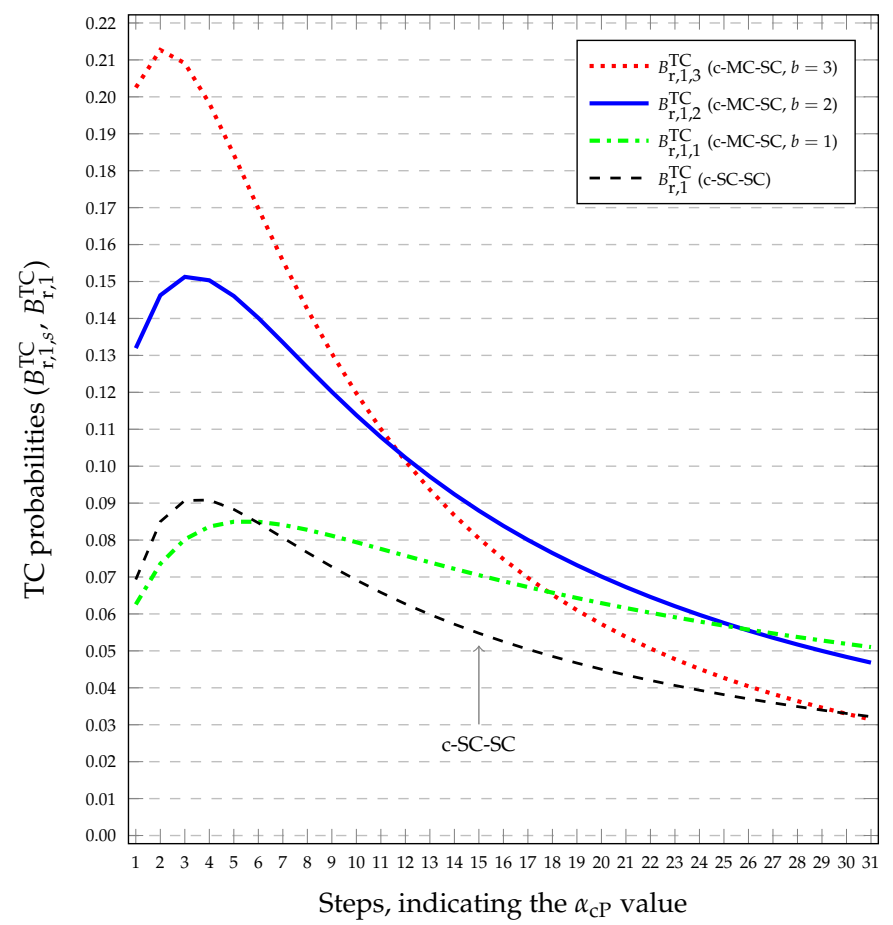

Figure 4. TC probabilities, $B_{\mathrm{r}, 1, \mathrm{~T}}^{\mathrm{TC}}(s=1,2,3)$, for all service-classes in the 1st RRH (c-MC-SC vs. c-SC-SC).

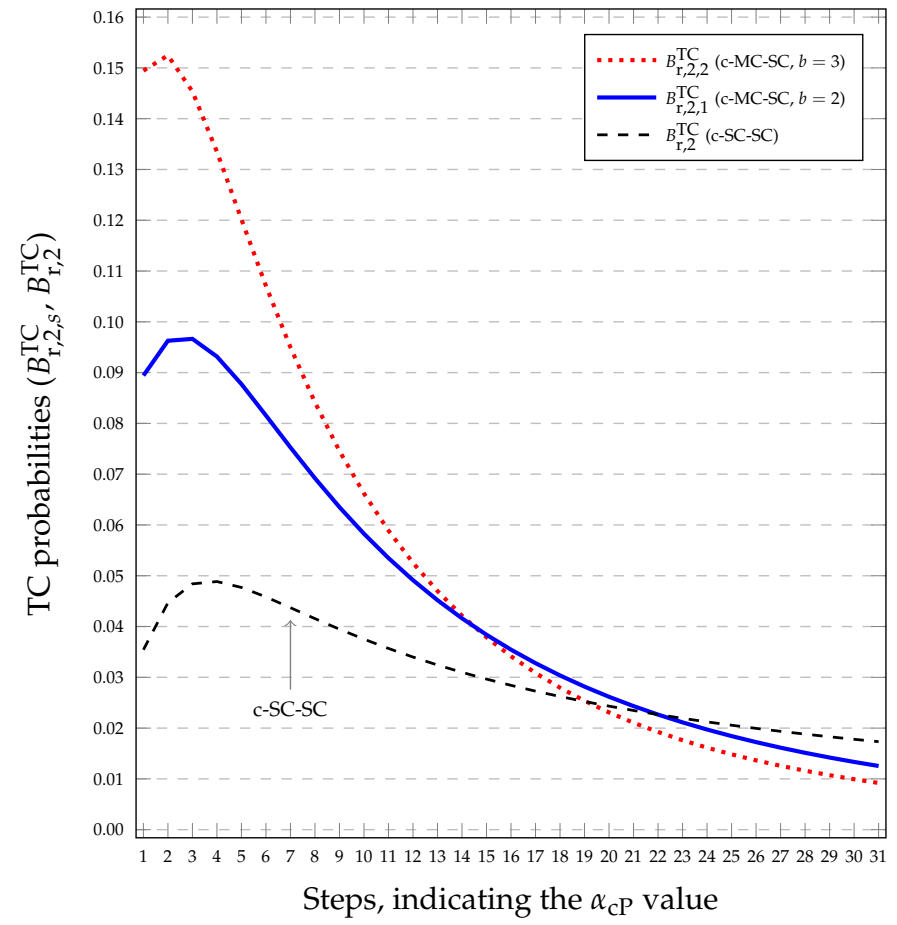

Figure 5. TC probabilities, $B_{\mathrm{r}, 2, S}^{\mathrm{TC}}(s=1,2)$, for all service-classes in the 2nd RRH (c-MC-SC vs. c-SC-SC). 


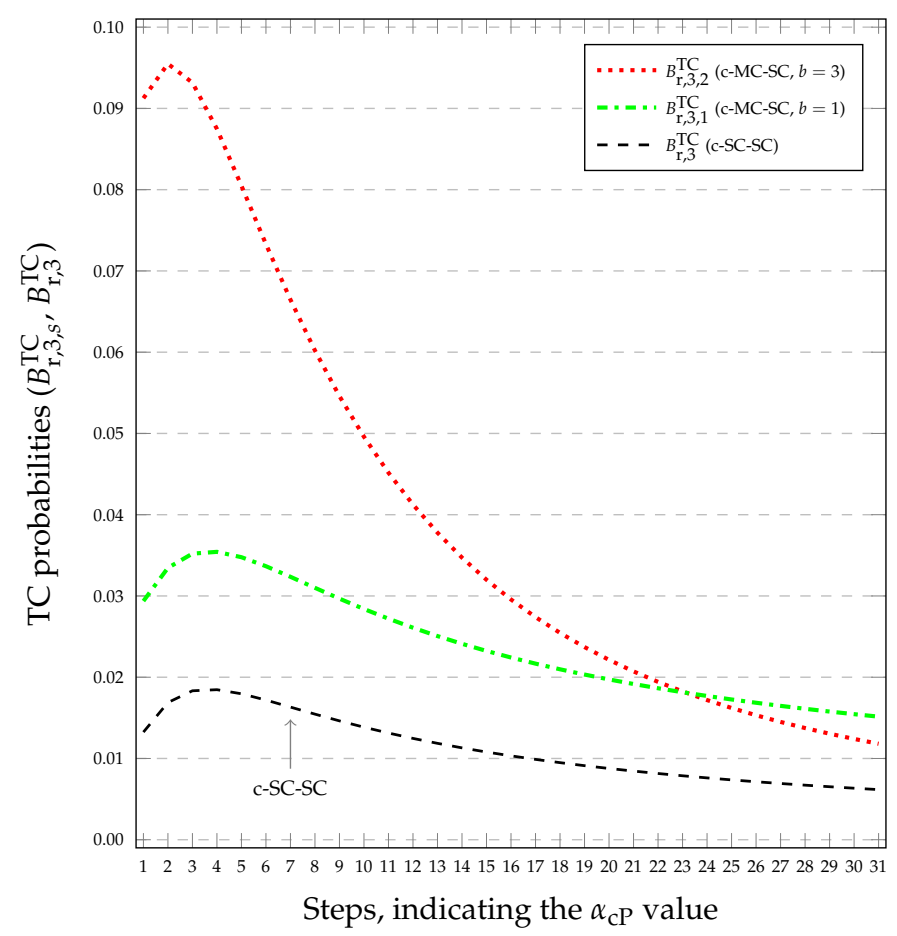

Figure 6. TC probabilities, $B_{\mathrm{r}, 3, s}^{\mathrm{TC}}(s=1,2)$, for all service-classes in the 3rd RRH (c-MC-SC vs. c-SC-SC).

In Figures 7-9, we consider again the 1st (see Figure 7), the 2nd (see Figure 8) and the 3rd RRH (see Figure 9) and present the corresponding total TC probabilities (calculated according to , (17), (18) and (30)). Based on Figures 7-9, we conclude that: (a) the increase in the incoming traffic-load leads to an increase in the total TC probabilities and (c) the existing single service model cannot provide TC probabilities close to the proposed multiservice model.

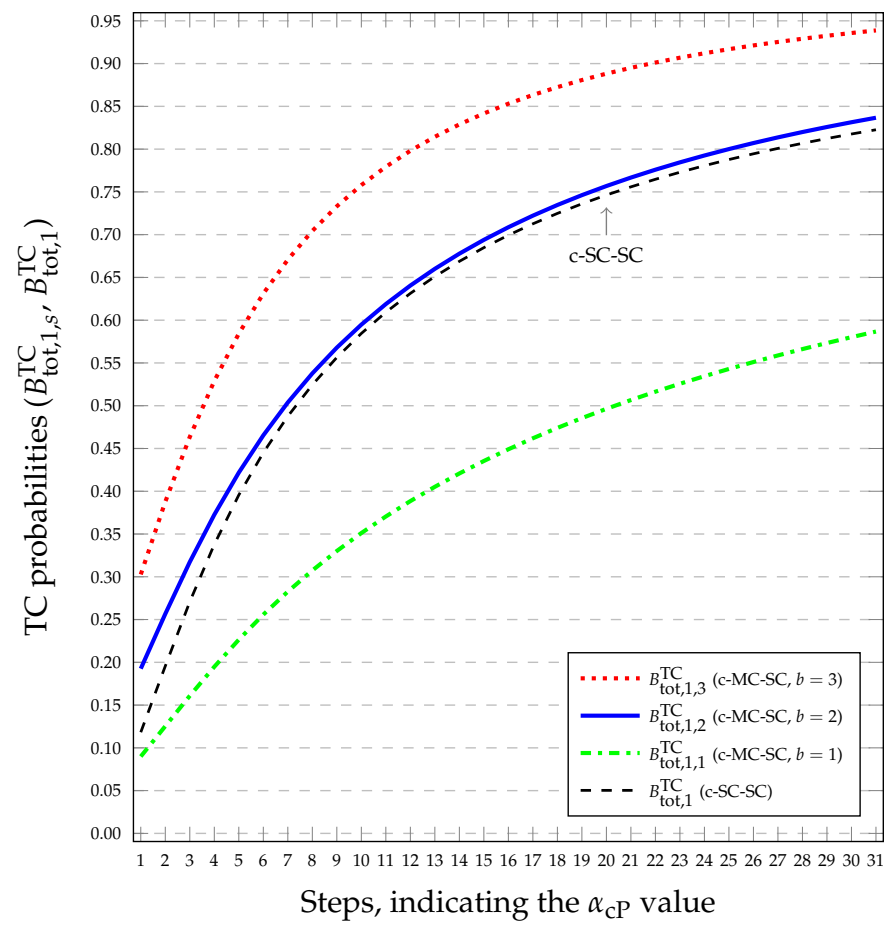

Figure 7. Total TC probabilities, $B_{\text {tot }, 1, s}^{\mathrm{TC}}(s=1,2,3)$, for all service-classes in the 1st RRH (c-MC-SC vs. c-SC-SC). 


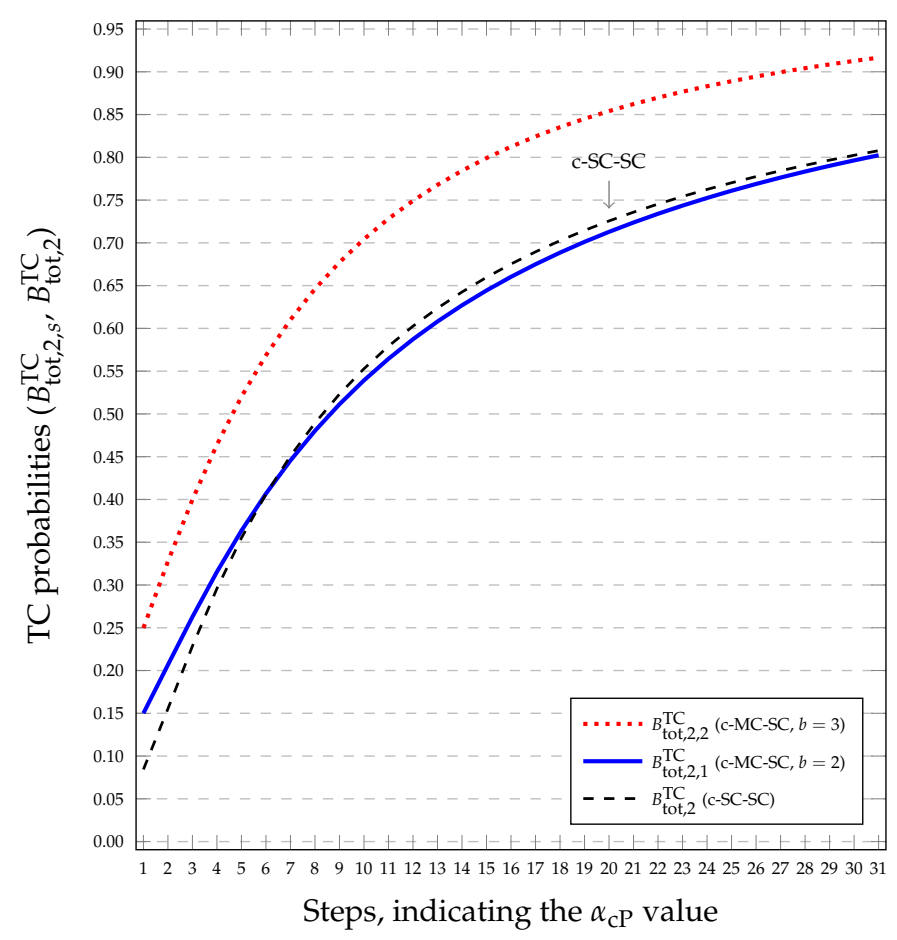

Figure 8. Total TC probabilities, $B_{\text {tot } 2, s}^{\mathrm{TC}}(s=1,2)$, for of all service-classes in the 2nd RRH (c-MC-SC vs. c-SC-SC).

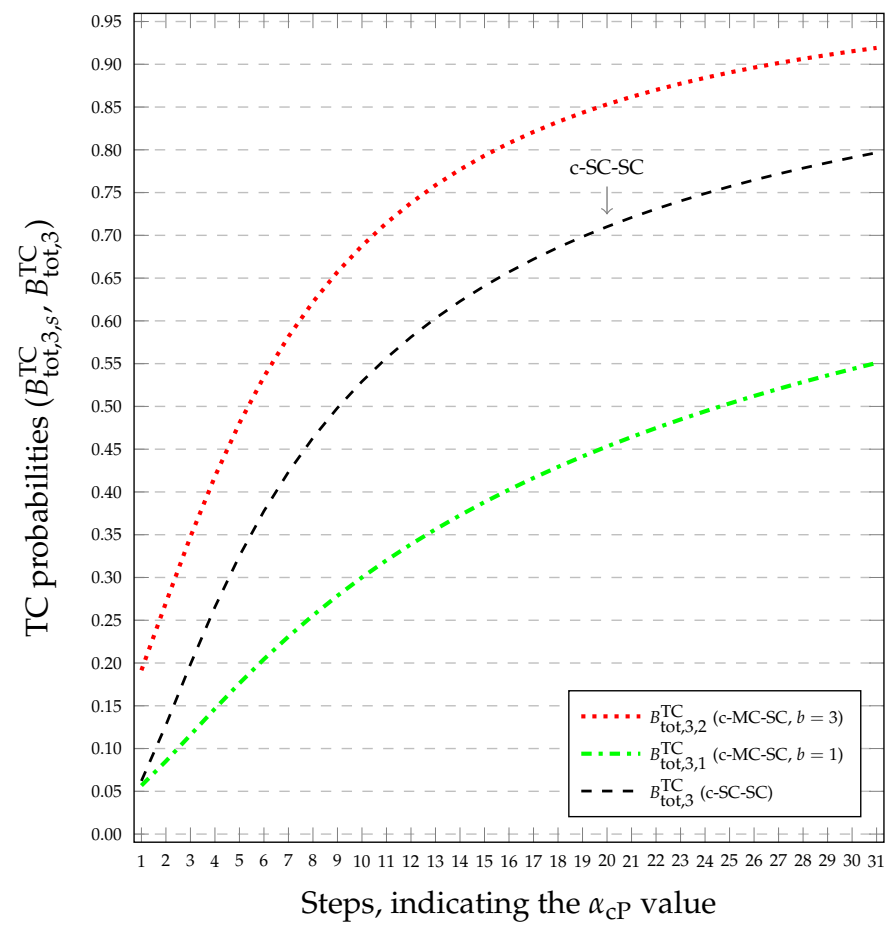

Figure 9. Total TC probabilities, $B_{\text {tot }, 3, s}^{\mathrm{TC}}(s=1,2)$, for all service-classes in the 3rd RRH (c-MC-SC vs. c-SC-SC).

\section{The Proposed Generalized c-MC-MC Model}

The generalized c-MC-MC model extends the proposed c-MC-SC model by considering the option that RRHs can form more than one cluster, where a cluster contains RRHs of the same capacity. Because of this, the generalized model supports RRHs of different RRU capacities. 


\subsection{The Analytical Model}

Figure 10 presents the network under consideration. Based on Figure 10, we see the $V$ different clusters of RRHs, which are separated from the T CRUs. Cluster $v(v=1, \ldots, V)$ includes $R_{v}$ RRHs of capacity $U_{v}$ RRUs.

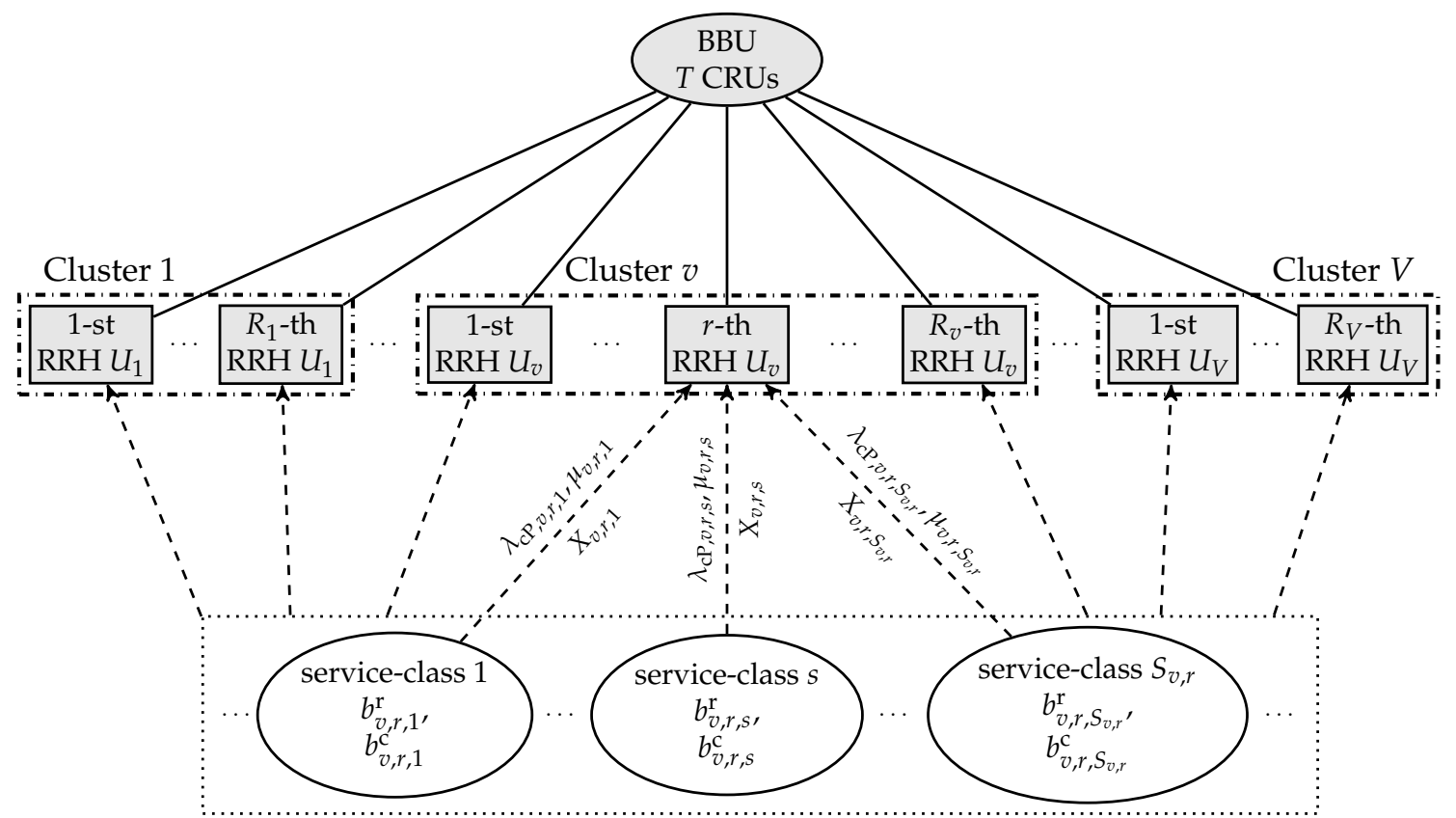

Figure 10. The extended proposed c-MC-MC model.

Considering cluster $v$, the $r$-th RRH $\left(r=1, \ldots, R_{v}\right)$ has the responsibility to serve compound Poisson traffic from $S_{v, r}$ service-classes. A batch of service-class $s\left(s=1, \ldots, S_{v, r}\right)$ calls arrives at the $v, r$-th RRH with an arrival rate of $\lambda_{\mathrm{cP}, v, r, s}$. Let $X_{v, r, s}$ be the random variable that expresses the batch size, then $P\left(X_{v, r, s}=x\right)$ is the probability that $x$ calls are included in a batch. Each of the $x$ calls does not depend on the remaining calls. Because of this and based on the availability of RRUs and CRUs, some calls can be accepted for service and the remaining calls (of the same batch) can be blocked and lost. A call of service-class $s$ of the $v, r$-th RRH requests $b_{v, r, s}^{\mathrm{r}}$ RRUs together with $b_{v, r, s}^{\mathrm{c}}$ CRUs. An accepted call remains in the $v, r$-th RRH for an exponentially distributed service time with mean $\mu_{v, r, s}^{-1}$ if the occupied RRUs in the $v, r$-th RRH are not greater than $U_{v}-b_{v, r, s}^{\mathrm{r}}$ and the occupied CRUs are not greater than $T-b_{v, r, s}^{\mathrm{c}}$. Otherwise, call blocking occurs.

Let $n_{v, r, s} \geq 0$ be the calls of service-class $s\left(s=1, \ldots, S_{v, r}\right)$ in the $v, r$-th RRH. Then, the corresponding vector for all RRHs is $\boldsymbol{n}=\left(n_{1,1,1}, \ldots, n_{1,1, S_{1,1}}, \ldots, n_{v, r, 1}, \ldots, n_{v, r, s}, \ldots\right.$, $\left.n_{v, r, S_{v, r}} \ldots, n_{V, R_{V}, 1} \ldots, n_{V, R_{V}, S_{V, R_{V}}}\right)$ and $\Omega$ can be given by:

$$
\boldsymbol{\Omega}=\left\{\boldsymbol{n}: n_{v, r, s} \geq 0, \quad \sum_{s=1}^{S_{v, r}} n_{v, r, s} b_{v, r, s}^{\mathrm{r}} \leq U_{v}, \quad \sum_{v=1}^{V} \sum_{r=1}^{R_{v}} \sum_{s=1}^{S_{v, r}} n_{v, r, s} b_{v, r, s}^{\mathrm{c}} \leq T\right\} .
$$

The analysis of the extended c-MC-MC model follows the same pattern as the c-MC-SC model. That is, we show that a form of local flow balance exists in the model that leads to a PFS for the probability distribution $P_{\mathrm{CP}}(\boldsymbol{n})$.

More specifically, for state $\boldsymbol{n}$ we denote the level $L_{\boldsymbol{n}}^{(v, r, s)}$, which separates $\boldsymbol{n}$ from $\boldsymbol{n}_{v, r, s}^{+}=\left(n_{1,1,1}, \ldots, n_{1,1, S_{1,1}} \ldots, n_{v, r, 1}, \ldots, n_{v, r, s}+1, \ldots, n_{v, r, S_{v, r}} \ldots, n_{V, R_{V}, 1} \ldots, n_{V, R_{V}, S_{V, R_{V}}}\right)$. To cross this level it is necessary to have a service-class $s$ batch arrival in the $v, r$-th RRH or a service-class $s$ call departure. The latter takes place when a call completes its service in the 
$v, r$-th RRH. Consider initially the case of a batch arrival. Then, the "upward" probability flow across $L_{n}^{(v, r, s)}$ is expressed by:

$$
p^{(\mathrm{upw})}\left(L_{n}^{(v, r, s)}\right)=\sum_{w=0}^{n_{v, r, s}} P_{\mathrm{cP}}\left(\boldsymbol{n}_{v, r, s}^{(-w)}\right) \lambda_{\mathrm{cP}, v, r, s} P\left(X_{v, r, s}>w\right),
$$

where $\quad \boldsymbol{n}_{v, r, s}^{-w}=\left(n_{1,1,1}, \ldots, n_{1,1, S_{1,1}}, \ldots, n_{v, r, 1}, \ldots, n_{v, r, s}-w, \ldots, n_{v, r, S_{v, r}}, \ldots, n_{V, R_{V}, 1}, \ldots\right.$, $\left.n_{V, R_{V}, S_{V, R_{V}}}\right)$ and $P_{\mathrm{CP}}\left(\boldsymbol{n}_{v, r, s}^{(-w)}\right)$ is the corresponding steady state probability.

Consider now the service completion of a call of service-class $s$ in the $v, r$-th RRH. In that case, the "downward" probability flow across $L_{n}^{(v, r, s)}$ is:

$$
p^{(\mathrm{dnw})}\left(L_{\boldsymbol{n}}^{(v, r, s)}\right)=\left(n_{v, r, s}+1\right) \mu_{v, r, s} P_{\mathrm{cP}}\left(\boldsymbol{n}_{v, r, s}^{+}\right)
$$

Via (23) and (24), we obtain the local balance equation across $L_{n}^{(v, r, s)}$ :

$$
\begin{aligned}
p^{(\mathrm{upw})}\left(L_{\boldsymbol{n}}^{(v, r, s)}\right) & =p^{(\mathrm{dnw})}\left(L_{n}^{(v, r, s)}\right) \text { or } \\
\sum_{w=0}^{n_{v, r, s}} P_{\mathrm{cP}}\left(\boldsymbol{n}_{v, r, s}^{(-w)}\right) \lambda_{\mathrm{cP}, v, r, s} P\left(X_{v, r, s}>w\right) & =\left(n_{v, r, s}+1\right) \mu_{v, r, s} P_{\mathrm{cP}}\left(\boldsymbol{n}_{v, r, s}^{+}\right) .
\end{aligned}
$$

Apart from (25), we also consider (26), which expresses the global balance equation for $n$ and service-class $v, r, s$ :

$$
p^{(\mathrm{upw})}\left(L_{\boldsymbol{n}_{v, r, s}^{-}}^{(v, r, s)}\right)+p^{(\mathrm{dnw})}\left(L_{\boldsymbol{n}}^{(v, r, s)}\right)=p^{(\mathrm{upw})}\left(L_{\boldsymbol{n}}^{(v, r, s)}\right)+p^{(\mathrm{dnw})}\left(L_{\boldsymbol{n}_{v, r, s}^{-}}^{(v, r, s)}\right),
$$

where $\boldsymbol{n}_{v, r, s}^{-} \equiv \boldsymbol{n}_{v, r, s}^{(-1)}$.

Summing over all $S_{v, r}$ in all RRHs leads to the global balance equation for state $n$ :

$$
\sum_{v=1}^{V} \sum_{r=1}^{R_{v}} \sum_{s=1}^{S_{v, r}}\left[p^{(\mathrm{upw})}\left(L_{\boldsymbol{n}_{v, r, s}^{-}}^{(v, r, s)}\right)+p^{(\mathrm{dnw})}\left(L_{n}^{(v, r, s)}\right)\right]=\sum_{v=1}^{V} \sum_{r=1}^{R_{v}} \sum_{s=1}^{S_{v, r}}\left[p^{(\mathrm{upw})}\left(L_{\boldsymbol{n}}^{(v, r, s)}\right)+p^{(\mathrm{dnw})}\left(L_{\boldsymbol{n}_{v, r}^{-}, s}^{(v, r, s)}\right)\right]
$$

Equations (26) and (27) can be satisfied by the PFS:

$$
P_{\mathrm{CP}}(\boldsymbol{n})=\frac{1}{N} \prod_{v=1}^{V} \prod_{r=1}^{R_{v}} \prod_{s=1}^{S_{v, r}} P_{v, r, s}\left(n_{v, r, s}\right)
$$

where $N=\sum_{n \in \Omega} \prod_{v=1}^{V} \prod_{r=1}^{R_{v}} \prod_{s=1}^{S_{v, r}} P_{v, r, s}\left(n_{v, r, s}\right)$ and

$$
P_{v, r, s}(n)=\left\{\begin{array}{ll}
\frac{\alpha_{\mathrm{cP}, v, r, s}}{n} \sum_{x=1}^{n} P_{v, r, s}(n-x) P\left(X_{v, r, s}>x-1\right), & n \geq 1 \\
1, & n=0
\end{array},\right.
$$

where $\alpha_{\mathrm{cP}, v, r, s}=\lambda_{\mathrm{cP}, v, r, s} / \mu_{v, r, s}$.

Having computed $P_{\mathrm{CP}}(\boldsymbol{n})$, we calculate the service-class $s$ TC probabilities in the $v, r$-th RRH: (a) due to the unavailability of RRUs, $B_{\mathrm{r}, v, r, s}^{\mathrm{TC}}$ and (b) due to the unavailability of CRUs,

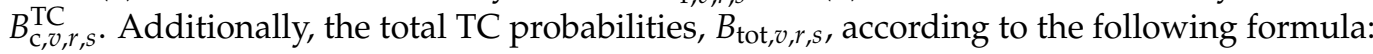

$$
B_{\mathrm{tot}, v, r, \mathrm{~s}}^{\mathrm{TC}}=B_{\mathrm{c}, v, r, s}^{\mathrm{TC}}+B_{\mathrm{r}, v, r, s}^{\mathrm{TC}}
$$

The values of $B_{\text {tot }, v, r, s}^{\mathrm{TC}}$ are obtained either via a brute force method (that can be adopted for small (tutorial) examples) or via an algorithm based on successive convolutions described in the next two subsections. 


\subsection{The Brute Force Method}

The values of $B_{\mathrm{r}, v, r, s}^{\mathrm{TC}}$ are given by:

$$
B_{\mathrm{r}, v, r, \mathrm{~s}}^{\mathrm{TC}}=\sum_{\boldsymbol{n} \in \mathbf{\Omega}_{v, r, s}^{U_{v},<T}} P_{\mathrm{cP}}(\boldsymbol{n}),
$$

where $\boldsymbol{\Omega}_{v, r, s}^{U_{v,}<T}=\left\{\boldsymbol{\Omega}_{v, r, s}^{U_{v}} \cap \boldsymbol{\Omega}_{v, r, s}^{<T}\right\}, \boldsymbol{\Omega}_{v, r, s}^{U_{v}}=\left\{\boldsymbol{n}: U_{v}-b_{v, r, s}^{\mathrm{r}}<\sum_{z=1}^{S_{v, r}} n_{v, r, z} b_{v, r, z}^{\mathrm{r}} \leq U_{v}\right\}$, $\boldsymbol{\Omega}_{v, r, s}^{<T}=\left\{\boldsymbol{n}: \sum_{x=1}^{V} \sum_{y=1}^{R_{x}} \sum_{z=1}^{S_{x, y}} n_{x, y, z} b_{x, y, z}^{\mathrm{c}} \leq T-b_{v, r, s}^{\mathrm{c}}\right\}$. Note that the set $\boldsymbol{\Omega}_{v, r, s}^{U_{v},<T}$ includes only the blocking states that express the lack of RRUs.

On the same hand, by denoting $\boldsymbol{\Omega}_{v, r, s}^{=T}=\left\{\boldsymbol{n}: T-b_{v, r, s}^{\mathrm{c}}<\sum_{x=1}^{V} \sum_{y=1}^{R_{x}} \sum_{z=1}^{S_{x, y}} n_{x, y, z} b_{x, y, z}^{\mathrm{c}}\right.$ $\leq T\}$, the set of blocking states that express the lack of CRUs together with the blocking states that express both insufficient RRUs and CRUs, we compute the TC probability of service-class $v, r, s$ calls due to lack of CRUs via:

$$
B_{\mathrm{c}, v, r, s}^{\mathrm{TC}}=\sum_{\boldsymbol{n} \in \mathbf{\Omega}_{v, r, s}^{=T}} P_{\mathrm{cP}}(\boldsymbol{n})
$$

To circumvent the mandatory enumeration/processing of $\Omega$, the following convolution algorithm can be adopted.

\subsection{The Proposed Convolution Algorithm for the c-MC-MC Model}

To apply the convolution algorithm, we assume that $b_{v, r, s}^{\mathrm{r}}=b_{v, r, s}^{\mathrm{c}}$. The algorithm consists of the following steps:

Step 1

We start by determining the occupancy distribution for each service-class $s$ in the $v, r$-th RRH $\left(s=1, \ldots, S_{v, r}, r=1, \ldots, R_{v}, v=1, \ldots, V\right), q_{v, r, s}(j)$, under the assumption that the $v, r$-th RRH services only service-class $s$ and $q_{v, r, s}(0)=1$ :

$$
q_{v, r, s}(j)=\frac{\alpha_{\mathrm{cP}, v, r, s}}{i} \sum_{x=1}^{i} q_{v, r, s}\left(j-x b_{v, r, s}^{\mathrm{r}}\right) P\left(X_{v, r, s}>x-1\right)
$$

where $i=j / b_{v, r, s}^{\mathrm{r}}$ expresses the calls of service-class $s$ in the $v, r$-th RRH and $j \in\left\{i b_{v, r, s}^{\mathrm{r}}\right.$ : $\left.i=0, \ldots,\left\lfloor U_{v} / b_{v, r, s}^{\mathrm{r}}\right\rfloor\right\}$ the corresponding occupied RRUs.

Having computed $q_{v, r, s}^{\prime}(j)$, we continue with the computation of, $q_{v, r}$, by successive convolution of all $q_{v, r, s}, s=1, \ldots, s_{v, r}$ :

$$
q_{v, r}=q_{v, r, 1} * \ldots * q_{v, r, s} * \ldots * q_{v, r, S_{v, r}}
$$

where the operation between $q_{v, r, x} \equiv q_{a}$ and $q_{v, r, y} \equiv q_{b}$ applied on the domain $\left\{0, \ldots, U_{v}\right\}$ is given by (14).

The values of $q_{v, r}(j)$ are normalized with the constant $N_{v, r}=\sum_{j=0}^{U_{v}} q_{v, r}(j)$ and are denoted via $q_{v, r}^{\prime}(j)=q_{v, r}(j) / N_{v, r}$.

Step 2

Herein, we determine the occupancy distribution of all RRHs excluding the $v, r$-th one, as follows:

$$
Q_{\mathrm{cP},(-(v, r))}=q_{1,1}^{\prime} * \ldots * q_{v, r-1}^{\prime} * q_{v, r+1}^{\prime} * \ldots * q_{V, R_{V}}^{\prime} .
$$

The operation between $q_{x, z}^{\prime} \equiv q_{a}$ and $q_{y, w}^{\prime} \equiv q_{b}$ is given by (14) applied on the domain $\{0, \ldots, T\}$. 
Step 3

In this step, we determine $\left(Q_{\mathrm{cP},(-(1,1))} * q_{1,1}^{\prime}\right)(j)$ on the domain $\{0, \ldots, T\}$. This operation leads to the values of the computational occupancy distribution $Q_{\mathrm{cP}}(j)$, which is normalized via $N_{\mathrm{cP}}^{*}=\sum_{j=0}^{T} Q_{\mathrm{cP}}(j)$ :

$$
Q_{\mathrm{cP}}^{\prime}(j)=\frac{Q_{\mathrm{cP}}(j)}{N_{\mathrm{cP}}^{*}} .
$$

To determine $Q_{\mathrm{cP}}^{\prime}(j)$, we may focus on any of the $v, r$ RRHs since the convolution operation is commutative and associative.

Based on (36), we compute the TC probabilities due to the unavailability of CRUs (see (37)) and RRUs (see (38)):

$$
\begin{gathered}
B_{\mathrm{c}, v, r, s}^{\mathrm{TC}}=\sum_{j=T-b_{v, r, s}^{\mathrm{c}}+1}^{T} Q_{\mathrm{cP}}^{\prime}(j), \\
B_{\mathrm{r}, v, r, s}^{\mathrm{TC}}=\frac{1}{N_{\mathrm{cP}}^{*}} \sum_{x=U_{v}-b_{v, r, s}^{\mathrm{r}}+1}^{U_{v}} q_{v, r}^{\prime}(x) \sum_{y=x}^{T-b_{v, r, s}^{\mathrm{r}}} Q_{\mathrm{cP},(-(v, r))}\left(T-b_{v, r, s}^{\mathrm{r}}-y\right) .
\end{gathered}
$$

The values of CC probabilities for the service-class $v, r, s$ calls, $B_{v, r, s}^{\mathrm{CC}}$, can be calculated via:

$$
B_{v, r, s}^{\mathrm{CC}}=\frac{\alpha_{\mathrm{cP}, v, r, s} \bar{X}_{v, r, s}-\bar{n}_{v, r, s}}{\alpha_{\mathrm{cP}, v, r, s} \bar{X}_{v, r, s}},
$$

where $\bar{X}_{v, r, s}$ refers to the average size of service-class $s$ batches that arrive at the $v, r$-th RRH and can be computed via $\bar{X}_{v, r, s}=\sum_{x=1}^{\infty} x P\left(X_{v, r, s}=x\right)$, while $\bar{n}_{v, r, s}$ is the mean number of service-class $s$ calls in the $v, r$-th RRH and can be calculated via:

$$
\bar{n}_{v, r, s}=\frac{1}{N_{\mathrm{cP}}^{*}} \sum_{j=1}^{U_{v}} y_{v, r, s}(j) q_{v, r}^{\prime}(j) \sum_{l=0}^{T-j} Q_{\mathrm{cP},(-(v, r))}(l),
$$

where $y_{z . m, k}(j)$ is the average number of service-class $s$ calls of the $v, r$-th RRH in state $j$ and can be obtained via:

$$
y_{v, r, s}(j)=\frac{\alpha_{\mathrm{cP}, v, r, s}}{q_{v, r}^{\prime}(j)} \sum_{l=1}^{\left\lfloor j / b_{v, r, s}^{\mathrm{r}}\right\rfloor} q_{v, r}^{\prime}\left(j-l b_{v, r, s}^{\mathrm{r}}\right) P\left(X_{v, r, s}>l-1\right) .
$$

\section{Conclusions}

Two new loss models that consider multirate traffic are proposed in this paper, namely the c-MC-SC and the c-MC-MC models, in order to analyze a C-RAN that accommodates different service-classes whose calls arrive in the system via a compound PP. The first model considers the case of RRHs that form a single cluster, while the second model considers the more general case of having many clusters of RRHs. We proved that the steady-state probabilities possess a PFS for both loss models, and additionally, we proposed algorithms based on successive convolutions for the efficient and accurate computation of TC and CC probabilities.

As future work, we intend to include in these models the important cases of elastic and adaptive service-classes. The main characteristic of such service-classes is the fact that the bandwidth (resource units) occupied by in-service calls is not constant but may fluctuate between a maximum and a minimum value [59-61]. Because of this, the steady-state distribution cannot be described by a PFS [62], a fact that may require additional changes in our convolution algorithms. 


\begin{abstract}
Author Contributions: Conceptualization, I.-A.C., I.M., P.S. and M.L.; methodology, I.-A.C., I.M., P.S. and M.L.; software, I.-A.C. and I.M.; validation, I.-A.C. and I.M.; writing-original draft preparation, I.-A.C., I.M., P.S. and M.L.; writing-review and editing, I.-A.C., I.M., P.S. and M.L. All authors have read and agreed to the published version of the manuscript.
\end{abstract}

Funding: This research received no external funding.

Institutional Review Board Statement: Not applicable.

Informed Consent Statement: Not applicable.

Data Availability Statement: Not applicable.

Conflicts of Interest: The authors declare no conflict of interest.

\title{
References
}

1. Ericsson. Ericsson Mobility Report November 2021; Report; Ericsson: Stockholm, Sweden, 2021.

2. Checko, A.; Christiansen, H.L.; Yan, Y.; Scolari, L.; Kardaras, G.; Berger, M.S.; Dittmann, L. Cloud RAN for Mobile Networks-A Technology Overview. IEEE Commun. Surv. Tutor. 2015, 17, 405-426. [CrossRef]

3. Zhu, M.; Gu, J.; Zeng, X.; Yan, C.; Gu, P. Delay-Aware Energy-Saving Strategies for BBU Pool in C-RAN: Modeling and Optimization. IEEE Access 2021, 9, 63257-63266. [CrossRef]

4. Alimi, I.; Teixeira, A.; Monteiro, P. Toward an Efficient C-RAN Optical Fronthaul for the Future Networks: A Tutorial on Technologies, Requirements, Challenges, and Solutions. IEEE Commun. Surv. Tutor. 2018, 20, 708-769. [CrossRef]

5. Aqeeli, E.; Moubayed, A.; Shami, A. Power-Aware Optimized RRH to BBU Allocation in C-RAN. IEEE Trans. Wirel. Commun. 2018, 17, 1311-1322. [CrossRef]

6. Mohammedali, N.A.; Kanakis, T.; Agyeman, M.O.; Al-Sherbaz, A. A Survey of Mobility Management as a Service in Real-Time Inter/Intra Slice Control. IEEE Access 2021, 9, 62533-62552. [CrossRef]

7. Mukhlif, F.; Noordin, K.; Mansoor, A.; Kasirun, Z. Green transmission for C-RAN based on SWIPT in 5G: A review. Wirel. Netw. 2019, 25, 2621-2649. [CrossRef]

8. Wang, L.; Zhou, S. On the Fronthaul Statistical Multiplexing Gain. IEEE Commun. Lett. 2017, 21, 1099-1102. [CrossRef]

9. Larsen, L.; Checko, A.; Christiansen, H. A Survey of the Functional Splits Proposed for 5G Mobile Crosshaul Networks. IEEE Commun. Surv. Tutor. 2019, 21, 146-172. [CrossRef]

10. Ismail, T.; Mahmoud, H.H.M. Optimum Functional Splits for Optimizing Energy Consumption in V-RAN. IEEE Access 2020, 8, 194333-194341. [CrossRef]

11. Touati, H.; Castel-Taleb, H.; Jouaber, B.; Akbarzadeh, S. Split analysis and fronthaul dimensioning in 5G C-RAN to guarantee ultra low latency. In Proceedings of the 2020 IEEE 17th Annual Consumer Communications \& Networking Conference (CCNC), Las Vegas, NV, USA, 10-13 January 2020; pp. 1-4. [CrossRef]

12. Erazo-Agredo, C.C.; Garza-Fabre, M.; Calvo, R.A.; Diez, L.; Serrat, J.; Rubio-Loyola, J. Joint Route Selection and Split Level Management for 5G C-RAN. IEEE Trans. Netw. Serv. Manag. 2021, 18, 4616-4638. [CrossRef]

13. Ahmad, I.; Kumar, T.; Liyanage, M.; Okwuibe, J.; Ylianttila, M.; Gurtov, A. Overview of 5G Security Challenges and Solutions. IEEE Commun. Stand. Mag. 2018, 2, 36-43. [CrossRef]

14. Santoyo-González, A.; Cervelló-Pastor, C. Network-Aware Placement Optimization for Edge Computing Infrastructure under 5G. IEEE Access 2020, 8, 56015-56028. [CrossRef]

15. Hossain, M.F.; Mahin, A.U.; Debnath, T.; Mosharrof, F.B.; Islam, K.Z. Recent research in cloud radio access network (C-RAN) for 5G cellular systems-A survey. J. Netw. Comput. Appl. 2019, 139, 31-48. [CrossRef]

16. Taghizadeh, O.; Yang, T.; Mathar, R. Private Uplink Communication in C-RAN with Untrusted Radios. IEEE Trans. Veh. Technol. 2020, 69, 8034-8039. [CrossRef]

17. Dai, B.; Yu, W. Energy Efficiency of Downlink Transmission Strategies for Cloud Radio Access Networks. IEEE J. Sel. Areas Commun. 2016, 34, 1037-1050. [CrossRef]

18. Li, Y.; Jiang, T.; Luo, K.; Mao, S. Green Heterogeneous Cloud Radio Access Networks: Potential Techniques, Performance Trade-offs, and Challenges. IEEE Commun. Mag. 2017, 55, 33-39. [CrossRef]

19. Tian, B.; Zhang, Q.; Li, Y.; Tornatore, M. Joint Optimization of Survivability and Energy Efficiency in 5G C-RAN with mm-Wave Based RRH. IEEE Access 2020, 8, 100159-100171. [CrossRef]

20. Masoudi, M.; Lisi, S.S.; Cavdar, C. Cost-Effective Migration Toward Virtualized C-RAN with Scalable Fronthaul Design. IEEE Syst. J. 2020, 14, 5100-5110. [CrossRef]

21. Opadere, J.; Liu, Q.; Han, T.; Ansari, N. Energy-Efficient Virtual Radio Access Networks for Multi-Operators Cooperative Cellular Networks. IEEE Trans. Green Commun. Netw. 2019, 3, 603-614. [CrossRef]

22. Kim, D.; Sung, K.W.; Koh, J.; Kang, J. On the benefit of inter-operator cooperation in C-RAN. IET Commun. 2021, 15, 191-199. [CrossRef]

23. Liu, J.; Zhou, S.; Gong, J.; Niu, Z.; Xu, S. On the statistical multiplexing gain of virtual base station pools. In Proceedings of the 2014 IEEE Global Communications Conference, Austin, TX, USA, 8-12 December 2014; pp. 2283-2288. [CrossRef] 
24. Avramova, A.; Christiansen, H.; Iversen, V. Cell Deployment Optimization for Cloud Radio Access Networks using Teletraffic Theory. In Proceedings of the Fifth International Conference on Advanced Communications and Computation, Haryana, India, 21-22 February 2015; pp. 96-101.

25. Checko, A.; Avramova, A.P.; Berger, M.S.; Christiansen, H.L. Evaluating C-RAN fronthaul functional splits in terms of network level energy and cost savings. J. Commun. Netw. 2016, 18, 162-172. [CrossRef]

26. Liu, J.; Zhou, S.; Gong, J.; Niu, Z.; Xu, S. Statistical Multiplexing Gain Analysis of Heterogeneous Virtual Base Station Pools in Cloud Radio Access Networks. IEEE Trans. Wirel. Commun. 2016, 15, 5681-5694. [CrossRef]

27. Chousainov, I.A.; Moscholios, I.; Sarigiannidis, P.; Kaloxylos, A.; Logothetis, M. An analytical framework of a C-RAN supporting random, quasi-random and bursty traffic. Comput. Netw. 2020, 180, 107410. [CrossRef]

28. Chousainov, I.A.; Moscholios, I.D.; Sarigiannidis, P.G. Congestion Probabilities in a Multi-Cluster C-RAN Servicing a Mixture of Traffic Sources. Electronics 2020, 9, 2120. [CrossRef]

29. Chousainov, I.A.; Moscholios, I.; Kaloxylos, A.; Logothetis, M. Performance Evaluation of a C-RAN Supporting Quasi-Random Traffic. In Proceedings of the 2019 International Conference on Software, Telecommunications and Computer Networks (SoftCOM), Split, Croatia, 19-21 September 2019; pp. 1-6. [CrossRef]

30. Chousainov, I.A.; Moscholios, I.; Sarigiannidis, P.; Kaloxylos, A.; Logothetis, M. An Analytical Framework of a C-RAN Supporting Bursty Traffic. In Proceedings of the ICC 2020-2020 IEEE International Conference on Communications (ICC), Dublin, Ireland, 7-11 June 2020; pp. 1-6. [CrossRef]

31. Stasiak, M.; Głąbowski, M.; Wisniewski, A.; Zwierzykowski, P. Modeling and Dimensioning of Mobile Networks: From GSM to LTE; John Wiley: Hoboken, NJ, USA, 2011. [CrossRef]

32. Moscholios, I.; Logothetis, M. Efficient Multirate Teletraffic Loss Models beyond Erlang; JohnWiley \& IEEE Press: Hoboken, NJ, USA, 2019. [CrossRef]

33. Chousainov, I.A.; Moscholios, I.; Kaloxylos, A.; Logothetis, M. Performance Evaluation in Single or Multi-Cluster C-RAN Supporting Quasi-Random Traffic. J. Commun. Softw. Syst. 2020, 16, 170-179. [CrossRef]

34. Głąbowski, M.; Kaliszan, A.; Stasiak, M. Modelling overflow systems with distributed secondary resources. Comput. Netw. 2016, 108, 171-183. [CrossRef]

35. Moscholios, I.D.; Vassilakis, V.G.; Logothetis, M.D.; Boucouvalas, A.C. State-Dependent Bandwidth Sharing Policies for Wireless Multirate Loss Networks. IEEE Trans. Wirel. Commun. 2017, 16, 5481-5497. [CrossRef]

36. Vassilakis, V.; Moscholios, I.; Logothetis, M. Efficient radio resource allocation in SDN/NFV based mobile cellular networks under the complete sharing policy. IET Netw. 2018, 7, 103-108. [CrossRef]

37. Hanczewski, S.; Stasiak, M.; Weissenberg, J. Queueing model of a multi-service system with elastic and adaptive traffic. Comput. Netw. 2018, 147, 146-161. [CrossRef]

38. Moscholios, I.; Vassilakis, V.; Sagias, N.; Logothetis, M. On Channel Sharing Policies in LEO Mobile Satellite Systems. IEEE Trans. Aerosp. Electron. Syst. 2018, 54, 1628-1640. [CrossRef]

39. Panagoulias, P.; Moscholios, I. Congestion probabilities in the X2 link of LTE Networks. Telecommun. Syst. 2019, 17, 585-599. [CrossRef]

40. Głąbowski, M.; Kaliszan, A.; Stasiak, M. A Palm-Jacobaeus Loss Formula for Multi-Service Systems with Separated Resources. Appl. Sci. 2020, 10, 4019. [CrossRef]

41. Głąbowski, M.; Sobieraj, M.; Stasiak, M.; Dominik Stasiak, M. Modeling of Clos Switching Structures with Dynamically Variable Number of Active Switches in the Spine Stage. Electronics 2020, 9, 1073. [CrossRef]

42. Panagoulias, P.; Moscholios, I.; Sarigiannidis, P.; Logothetis, M. Congestion probabilities in OFDM wireless networks with compound Poisson arrivals. IET Commun. 2020, 14, 674-681. [CrossRef]

43. Głąbowski, M.; Leitgeb, E.; Sobieraj, M.; Stasiak, M. Analytical Modeling of Switching Fabrics of Elastic Optical Networks. IEEE Access 2020, 8, 193462-193477. [CrossRef]

44. Głabowski, M.; Kmiecik, D.; Stasiak, M. On Increasing the Accuracy of Modeling Multi-Service Overflow Systems with Erlang-Engset-Pascal Streams. Electronics 2021, 10, 508. [CrossRef]

45. Chousainov, I.A.; Moscholios, I.D.; Sarigiannidis, P.; Logothetis, M.D. Multiservice Loss Models for Cloud Radio Access Networks. IEEE Access 2021, 9, 125109-125125. [CrossRef]

46. Chousainov, I.A.; Moscholios, I.; Sarigiannidis, P.; Logothetis, M. Multiservice Loss Models in Single or Multi-Cluster C-RAN Supporting Quasi-Random Traffic. Appl. Sci. 2021, 11, 8559. [CrossRef]

47. Mpantola, E.; Vardakas, J.; Louta, M. Performance evaluation of Cloud Radio Access Networks by jointly considering communicational and computational network resources. In Proceedings of the 2021 6th South-East Europe Design Automation, Computer Engineering, Computer Networks and Social Media Conference (SEEDA-CECNSM), Preveza, Greece, 24-26 September 2021; pp. 1-5. [CrossRef]

48. Kaufman, J. Blocking in a Shared Resource Environment. IEEE Trans. Commun. 1981, 29, 1474-1481. [CrossRef]

49. Kaufman, J. Blocking in a completely shared resource environment with state dependent resource and residency requirements. In Proceedings of the IEEE INFOCOM '92: The Conference on Computer Communications, Florence, Italy, 4-8 May 1992; Volume 3, pp. 2224-2232. [CrossRef]

50. Moscholios, I.D.; Logothetis, M.D.; Kokkinakis, G.K. Connection-dependent threshold model: A generalization of the Erlang multiple rate loss model. Perform. Eval. 2002, 48, 177-200. [CrossRef] 
51. Vassilakis, V.G.; Moscholios, I.D.; Logothetis, M.D. The extended connection-dependent threshold model for call-level performance analysis of multi-rate loss systems under the bandwidth reservation policy. Int. J. Commun. Syst. 2012, 25, 849-873. [CrossRef]

52. Moscholios, I.D.; Logothetis, M.D. The Erlang multirate loss model with Batched Poisson arrival processes under the bandwidth reservation policy. Comput. Commun. 2010, 33, S167-S179. [CrossRef]

53. Vardakas, J.S. Electric vehicles charging management in communication controlled fast charging stations. In Proceedings of the 2014 IEEE 19th International Workshop on Computer Aided Modeling and Design of Communication Links and Networks (CAMAD), Athens, Greece, 1-3 December 2014; pp. 115-119. [CrossRef]

54. Li, W.; Kouvatsos, D.D.; Fretwell, R.J. Towards time domain invariant QoS measures for queues with correlated traffic. J. Comput. Syst. Sci. 2015, 81, 132-144. [CrossRef]

55. Ezhilchelvan, P.; Mitrani, I. Multi-class Resource Sharing with Batch Arrivals and Complete Blocking. In Proceedings of the International Conference on Quantitative Evaluation of Systems, Berlin, Germany, 5-7 September 2017; Springer International Publishing: Cham, Switzerland, 2017; Volume 10503, pp. 157-169. [CrossRef]

56. Escheikh, M.; Tayachi, Z.; Barkaoui, K. Performability evaluation of server virtualized systems under bursty workload. IFACPapersOnLine 2018, 51, 45-50. [CrossRef]

57. Shahsavari, Y.; Shahhoseini, H.; Zhang, K.; Elbiaze, H. A Theoretical Model for Analysis of Firewalls under Bursty Traffic Flows. IEEE Access 2019, 7, 183311-183321. [CrossRef]

58. Harrison, P. A semi-product-form for a pair of queues with finite batches: Equilibrium state probabilities and response time densities. Perform. Eval. 2020, 143, 102120. [CrossRef]

59. Moscholios, I.D.; Vardakas, J.S.; Logothetis, M.D.; Boucouvalas, A.C. QoS guarantee in a batched poisson multirate loss model supporting elastic and adaptive traffic. In Proceedings of the International Conference on Communications (ICC), Ottawa, ON, Canada, 10-15 June 2012; pp. 1281-1286. [CrossRef]

60. Moscholios, I.D.; Logothetis, M.D.; Boucouvalas, A.C. Blocking probabilities of elastic and adaptive calls in the Erlang multirate loss model under the threshold policy. Telecommun. Syst. 2016, 62, 245-262. [CrossRef]

61. Hanczewski, S.; Stasiak, M.; Weissenberg, J. A Model of a System With Stream and Elastic Traffic. IEEE Access 2021, 9, 7789-7796. [CrossRef]

62. Stamatelos, G.; Koukoulidis, V. Reservation-based bandwidth allocation in a radio ATM network. IEEE/ACM Trans. Netw. 1997, 5, 420-428. [CrossRef] 